\title{
Measurement of antioxidant activity with trifluoperazine dihydrochloride radical cation
}

\author{
M.N. Asghar and I.U. Khan \\ Department of Chemistry, Government College University, Lahore, Pakistan \\ Correspondence to: I.U. Khan, Department of Chemistry, Government College University, Lower Mall, \\ Lahore-54000, Pakistan \\ E-mail: iukhan@gcu.edu.pk
}

\begin{abstract}
A novel, rapid and cost-effective trifluoperazine dihydrochloride (TFPH) decolorization assay is described for the screening of antioxidant activity. A chromogenic reaction between TFPH and potassium persulfate at low pH produces an orange-red radical cation with maximum absorption at $502 \mathrm{~nm}$ in its first-order derivative spectrum. TFPH was dissolved in distilled water to give a $100 \mathrm{mM}$ solution. The TFPH radical cation solution was made by reacting $0.5 \mathrm{~mL}$ of the solution with $\mathrm{K}_{2} \mathrm{~S}_{2} \mathrm{O}_{8}$ (final concentration: $0.1 \mathrm{mM}$ ) and diluting to $100 \mathrm{~mL}$ with $4 \mathrm{M} \mathrm{H}_{2} \mathrm{SO}_{4}$ solution. A linear inhibition of color production was observed with linearly increasing amounts of antioxidants, with correlation coefficients $\left(R^{2}\right)$ ranging from 0.999 to 0.983 . The antioxidant capacity of standard solutions of an antioxidant was evaluated by comparing with the inhibition curve using Trolox as the standard. Comparison of antioxidant capacity determined with this newly developed TFPH assay and with the well-known 2,2'azinobis-[3-ethylbenzthiazoline-6-sulfonic acid] (ABTS)-persulfate decolorization assay indicated the efficacy and sensitivity of the procedure. The proposed assay is less expensive (costs about US\$4 per 100 assays) and requires only 20 min for preparation of radical cation solution in comparison with ABTS assay, in which almost 12-16 $\mathrm{h}$ are required for preparation of a stable ABTS radical cation solution. The present assay has the advantage over ABTS assay that it can be used to measure the antioxidant activity of the samples, which are naturally found at a $\mathrm{pH}$ as low as 1 , because the radical cation itself has been stabilized at low $\mathrm{pH}$.
\end{abstract}

Key words: Trifluoperazine dihydrochloride; 2,2'-Azinobis-[3-ethylbenzthiazoline-6-sulfonic acid] (ABTS); Measurement of antioxidant activity; Trolox; Radical cation

Research supported by the Higher Education Commission of Pakistan under its Indigenous PhD 5000 Fellowship Program Batch-II (042-121235-PS2-286).

Received September 12, 2006. Accepted January 14, 2008

\section{Introduction}

The over-production of reactive oxygen species (ROS) has been reported to be implicated in the development of diabetes, arteriosclerosis, aging, and other degenerative diseases (1-5). The amplitude of oxidative stress is judged either by directly determining the strength of ROS or by evaluating the level of antioxidants present against ROS. Many electron transfer-based assays are being used to measure the reducing capacity of antioxidants (6-12). Depending upon the stability of radical cations and the nature of the samples, these assays are carried out at different
$\mathrm{pH}$. For example, the total phenol assay by the FolinCiocalteau reagent is performed under basic conditions, the trolox equivalent antioxidant capacity (TEAC) assay employs neutral conditions, whereas the ferric reducing antioxidant power assay is performed under acidic conditions. Different buffer systems have been used in order to obtain a medium in which the radical cation or the chromogen is stable. However, in addition to stabilizing the radical cation, the buffer systems by controlling the ratio of the protonated or deprotonated states of the antioxidants could result in an "induced antioxidant" activity.

The control of $\mathrm{pH}$ has been shown to be the important 
factor in the determination of the radical scavenging ability of anthocyanins and hydroxyflavones, since an increase in $\mathrm{pH}$ significantly increased the radical scavenging capacity of the antioxidants $(13,14)$. At high $\mathrm{pH}$, proton dissociation of the hydroxyl moiety of polyphenols enhances the reducing power of antioxidants, while at low $\mathrm{pH}$ the protonation suppresses the antioxidant power (15). The TEAC values which have so far been reported in the literature are mostly at $\mathrm{pH} 7.4$ and are not related to the TEAC values of polyphenols in their non-deprotonated state. Thus, in order to determine the antioxidant potential of polyphenolic compounds in their non-deprotonated state, there was the need to develop a decolorization assay in which the radical cation itself would be stabilized at low $\mathrm{pH}$.

Trifluoperazine dihydrochloride (TFPH) and other phenothiazine congeners are being extensively used as antipsychotic drugs for treating psychosis and behavioral disturbances. A broad spectrum of biological activity of phenothiazine drugs has been explored but their use for the measurement of antioxidant activity has not been described.

The present study describes an economical, sensitive and accurate decolorization assay based on the generation of a novel TFPH radical cation and its subsequent scavenging in the presence of antioxidants. In addition, the assay could also be used to estimate the intrinsic antioxidant activity of the samples, which are inherently found at low $\mathrm{pH}$.

\section{Material and Methods}

Trolox (6-hydroxy-2,5,7,8-tetramethylchroman-2-carboxylic acid) was obtained from Aldrich Chemical Co. (Gillingham, Dorset, UK) while the other standard antioxidants and TFPH were obtained from Fluka Chemicals (Basel, Switzerland). The working solutions of standards were prepared in methanol for trolox, gallic acid, 2,2'methylene bis(6-ter-butyl-4-methyl phenol), kaempferol, and cyanidin chloride and in twice-distilled water for reduced glutathione and ascorbic acid. The solutions were diluted in such a way as to fall within the activity range of the assay. Experiments were performed using the CECIL CE-7200 UV-Visible spectrophotometer (Cecil Instruments, Ltd., Cambridge, England).

Assay protocol: trifluoperazine dihydrochloride decolorization assay

A stock solution of $100 \mathrm{mM}$ TFPH was obtained by dissolving $0.480 \mathrm{~g} \mathrm{TFPH}$ in $10 \mathrm{~mL}$ distilled water. The solution can be stored at $4^{\circ} \mathrm{C}$ in the dark and can be used for at least 2 months. TFPH stock solution $(0.5 \mathrm{~mL})$ and 0.1
$\mathrm{mL} 100 \mathrm{mM} \mathrm{K}_{2} \mathrm{~S}_{2} \mathrm{O}_{8}$ were added to about $70 \mathrm{~mL} 4 \mathrm{M} \mathrm{H}_{2} \mathrm{SO}_{4}$ and the volume was completed to $100 \mathrm{~mL}$ with $4 \mathrm{M} \mathrm{H}_{2} \mathrm{SO}_{4}$. The solution was kept in the dark and the absorbance was monitored for about 15-20 min at $502 \mathrm{~nm}$ until it reached a constant value $(0.70 \pm 0.1$ absorbance units). Since TFPH and potassium persulfate react stoichiometrically at 1:0.5, the addition of potassium persulfate to the TFPH solution results in incomplete oxidation. At first, the intensity of the solution increases rapidly but then slows and finally becomes stable after about $15-20 \mathrm{~min}$. The solution in this form is stable for almost $2 \mathrm{~h}$ when stored in the dark at $25^{\circ} \mathrm{C}$. In order to study antioxidant activity, food extracts and plasma antioxidants (in water), and phenolic compounds (in ethanol) were diluted in such a way that after the addition of $10 \mu \mathrm{L}$ of sample to $3.49 \mathrm{~mL}$ of the TFPH radical cation solution they produced about $20-60 \%$ inhibition of the blank. Percent inhibition was determined as: \% inhibition $=\left(1-A_{f} / A_{o}\right) \times 100$, where $A_{o}$ is the absorbance at $502 \mathrm{~nm}$ of the unscavenged radical cation solution while $A_{f}$ is the absorbance after the addition of the antioxidant sample. Absorbance was monitored for $3 \mathrm{~min}$ after the addition of the antioxidant solution. All measurements were made in triplicate at each concentration of the standard and solvent samples, with an appropriate solvent blank being used for each assay. Percent inhibition was calculated and plotted as a function of the concentration of standard antioxidants. The TEAC of the samples ( $\mathrm{mmol} / \mathrm{L}$ ) was determined by comparing its percent inhibition with that of the standard curve for trolox.

Assay protocol: 2,2'-azinobis-[3-ethylbenzthiazoline-6sulfonic acid] decolorization assay

2,2'-Azinobis-[3-ethylbenzthiazoline-6-sulfonic acid] (ABTS) was dissolved in twice-distilled water to yield a $7 \mathrm{mM}$ solution. The ABTS radical cation solution was prepared by allowing the ABTS solution to react with the $\mathrm{K}_{2} \mathrm{~S}_{2} \mathrm{O}_{8}$ solution (final concentration: $2.45 \mathrm{mM}$ ) for $12-16 \mathrm{~h}$ in the dark at room temperature. For the determination of the antioxidant capacity of synthetic solutions of standard antioxidants and citrus juice, the ABTS solution was diluted with an appropriate solvent (methanol for lipophilic antioxidants and PBS for hydrophilic antioxidants) to an absorbance of $0.7 \pm 0.01$. The synthetic solutions of standard antioxidants were diluted in such a way that a quantity of $10 \mu \mathrm{L}$ of the sample would produce a 20 to $70 \%$ inhibition of absorbance when added to $2.99 \mathrm{~mL}$ ABTS working solution.

\section{Results and Discussion}

The trifluoperazine dihydrochloride decolorization assay The fact that some trace metals (e.g., $\mathrm{Mn}^{3+}, \mathrm{Co}^{3+}, \mathrm{Fe}^{3+}$, 
and other oxidants) and phenothiazine derivatives interact with each other was first indicated when it was observed that a thiazine drug (chlorpromazine) could markedly suppress the binding of divalent manganese by soluble proteins, etc. $(16,17)$. The chromogenic reaction product, formed as a result of univalent oxidation of phenothiazine ring-containing drugs, was confirmed to be a radical cation by the electrochemical and magnetic resonance data presented by Michaelis et al. (18). In the present study, a chromogenic reaction between trifluoperazine (phenothiazine) and potassium persulfate (oxidant) was developed and standardized in an effort to establish a decolorization assay for the estimation of antioxidant activity of a range of antioxidants and intrinsic antioxidant activity of samples which are naturally found at low $\mathrm{pH}$.

The structure of TFPH is shown in Figure 1. TFPH is soluble in water and its solubility can be ascribed primarily to protonation of the substituted amine side chain. In aqueous medium TFPH reacts with potassium persulfate, yielding an orange-red-colored product, which is metastable and photosensitive regardless of the nature of the oxidant. The color of the chromogen fades away within a few minutes and the product becomes colorless. The normal and first-order derivative spectra of the TFPH radical cation solution were recorded in $1-\mathrm{cm}$ silica cells at a fixed slit width of $2 \mathrm{~nm}$ over the wavelength range of 300$900 \mathrm{~nm}$ (Figure 2). The 1st derivative spectrum (Figure 2B) confirms the absorption maximum as $502 \mathrm{~nm}$.

Figure 2 shows that TFPH can react with different concentrations of potassium persulfate, showing a promising aspect of its determination through a calibration curve at $502 \mathrm{~nm}$. It is evident from Figure 3 that the UV-visible absorption spectrum of the reaction product of TFPH and persulfate is characteristically different from those of native TFPH and the bleached product of the reaction. Reportedly, the sulfur atom on the planar ring system of phenothiazine could donate a pair of electrons to the metals (oxidant) to produce a colorless sulfoxide product, as shown in Figure 1 (19).

Although the radical cation may be obtained from the reaction of TFPH and potassium persulfate at physiological $\mathrm{pH}$, it is not stable for a long period of time. The stability of semiquinone radicals that are Wurster type radicals depends mainly upon the extent of attainment of equivalent resonance (the resonance equivalent to the resonance found in a typical Wurster type radical). In the case of the TFPH radical cation, however, no immediate analogy with Wurster type radicals seems to be obvious. When the chromogenic reaction is carried out in an acid medium the stability of the radical cation increases. Figure 4 shows the effect of an aqueous medium of various acid strengths on the stability of the TFPH radical cation. It has been reported that phenothiazine sulfoxide, with its thioether site blocked by oxygen, could not be made to develop a

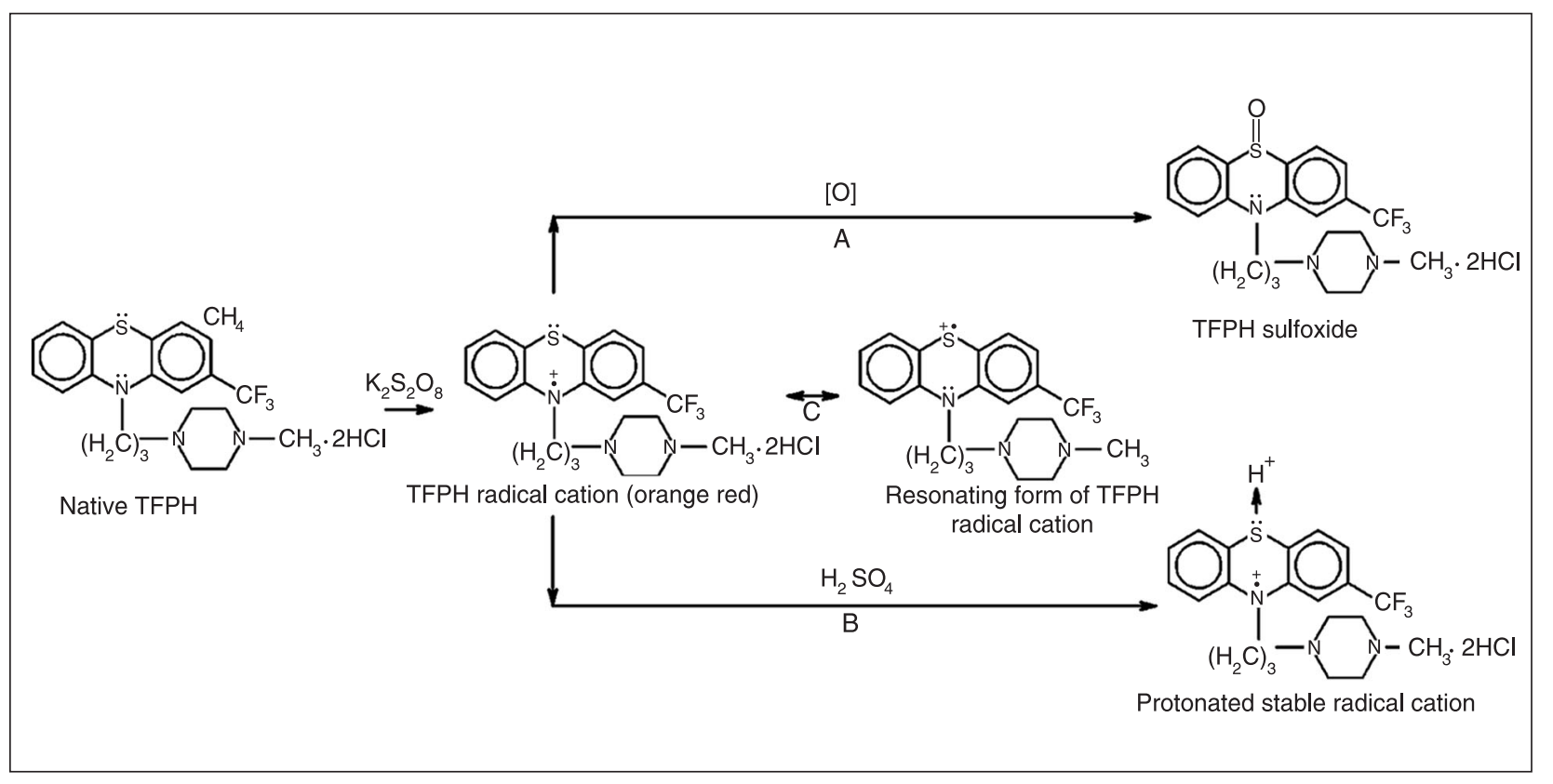

Figure 1. Structural representation showing the conversion of the trifluoperazine dihydrochloride (TFPH) radical cation to $A$, TFPH sulfoxide (in the absence of acid medium); $B$, protonated stable TFPH radical cation form (in acid medium), and $C$, its resonating form. 


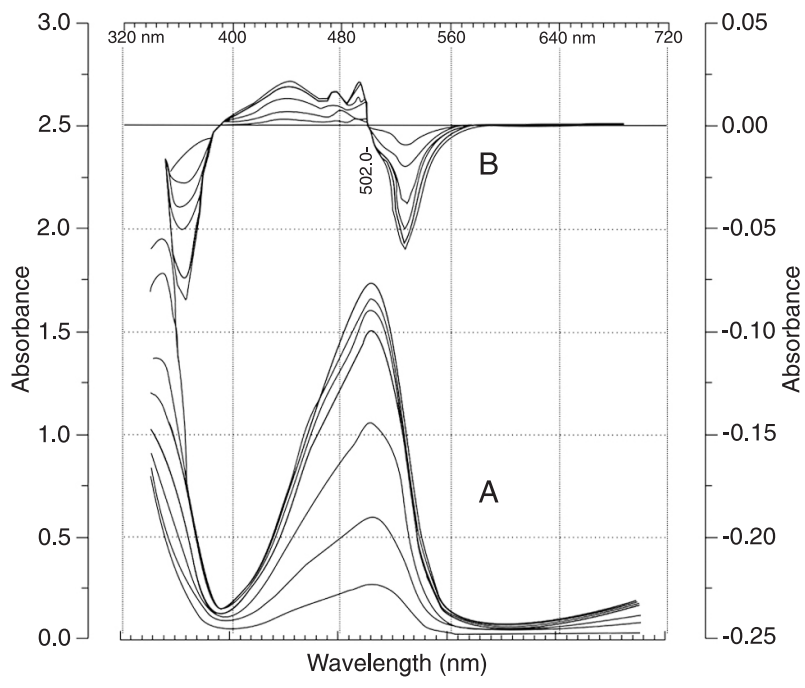

Figure 2. Overlayed normal (A) and 1st derivative of normal (B) absorption spectra of the colored reaction product from trifluoperazine dihydrochloride radical cation at different concentrations $(0.01-4 \mathrm{mM})$ of potassium persulfate.

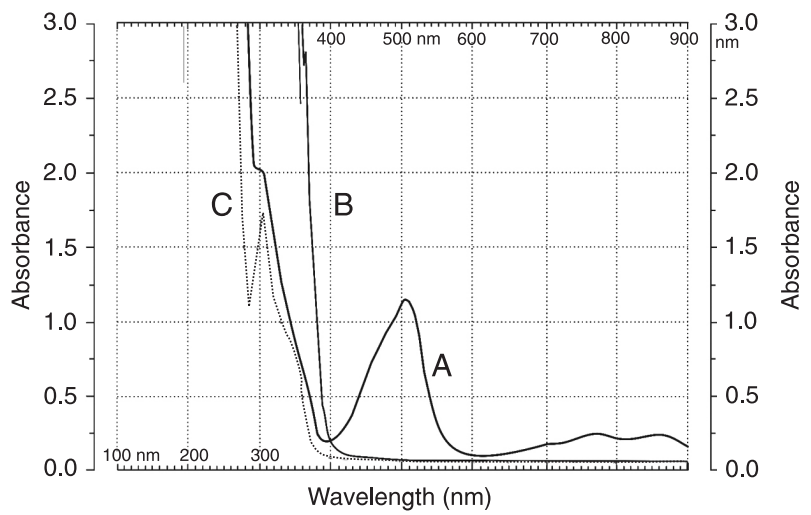

Figure 3. Overlayed normal absorption spectra of $A$, trifluoperazine dihydrochloride (TFPH) radical cation; $B$, native TFPH, and $C$, TFPH sulfoxide.

colored product with any of the oxidizing systems that are chromogenic with native phenothiazine (20). Likewise, it can easily be inferred that, in the case of TFPH, the high strength of acid may inhibit or retard the formation of sulfoxide by the protonation of the sulfur atom. Our results support this fact and a representation of the convergence of native TFPH to a radical cation and then to sulfoxide is given in Figure 1. At a concentration of about $4 \mathrm{M} \mathrm{H}_{2} \mathrm{SO}_{4}$ the radical is stable for almost $2 \mathrm{~h}$. The stability of the TFPH radical cation, although lacking any equivalence resonance or Wurster type resonance, can be attributed to resonance between a pair of limiting structures. For such resonance, no side chain in the phenothiazine ring is

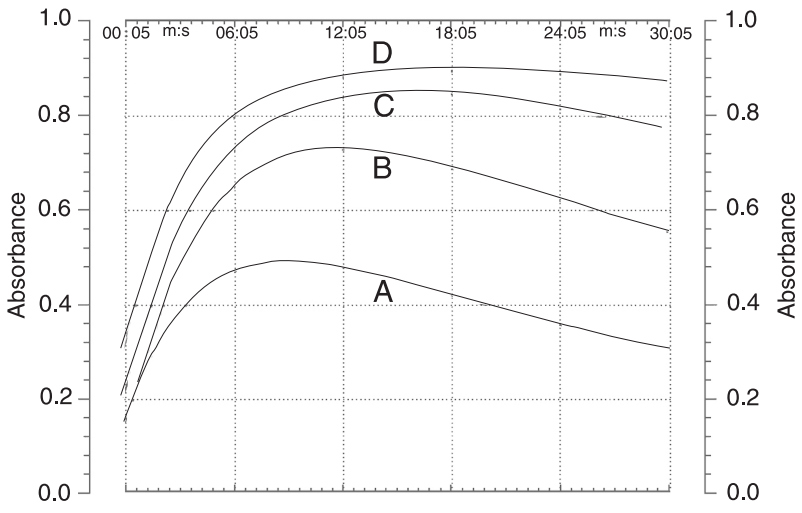

Figure 4. Time course of trifluoperazine dihydrochloride (TFPH) radical cation formation in aqueous medium of different acid strengths (A-D = 1-4 $\mathrm{M} \mathrm{H}_{2} \mathrm{SO}_{4}$ ). The final concentration of TFPH and $\mathrm{K}_{2} \mathrm{~S}_{2} \mathrm{O}_{8}$ was 0.5 and $0.1 \mathrm{mM}$, respectively, in all four cases.

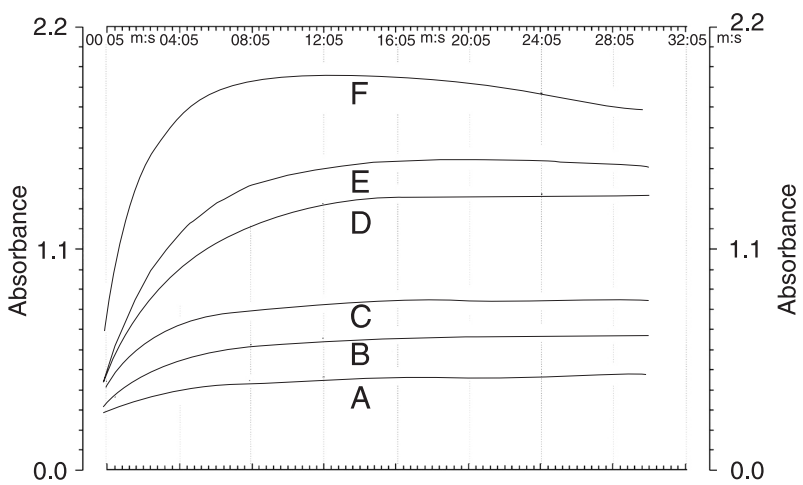

Figure 5. Time course of trifluoperazine dihydrochloride (TFPH) radical cation formation using $4 \mathrm{M} \mathrm{H}_{2} \mathrm{SO}_{4}$ and different concentrations of $\mathrm{K}_{2} \mathrm{~S}_{2} \mathrm{O}_{8}(\mathrm{~A}-\mathrm{F}=0.02,0.05,0.1,1,2,4 \mathrm{mM}$, respectively). The final concentration of TFPH was $0.5 \mathrm{mM}$ in all cases.

needed to form a fairly stable radical cation.

The effect of different concentrations of an oxidizing agent $\left(\mathrm{K}_{2} \mathrm{~S}_{2} \mathrm{O}_{8}\right)$ on the intensity and stability of the radical cation was studied (Figure 5). As the concentration of persulfate was increased, the time for the attainment of maximum absorption was decreased, with a concomitant decrease in the stability of the radical cation. Although curves $C, D$, and $E$ seem to be equally acceptable in terms of both stability and intensity, we preferred a minimum concentration i.e., $0.1 \mathrm{mM}$ (curve $\mathrm{C}$ ) of persulfate for the chromogenic reaction in order to avoid presence of excess $\mathrm{K}_{2} \mathrm{~S}_{2} \mathrm{O}_{8}$ which might interfere with the reaction between antioxidant and TFPH radical cation, leading to erroneous 
results.

It has been demonstrated that thiazines and oxazines are converted to semiquinone radicals as intermediate reduction products before they are converted to their sulfoxides (18). Following the same pattern, the semiquinonelike radicals formed could be proposed as intermediate reduction products for TFPH (Figure 1). This idea was confirmed by reacting the TFPH radical cation with reduced glutathione, ascorbic acid, gallic acid, and other antioxidants, which efficiently inhibited and reversed the chromogenic reaction.

The inhibition or scavenging of the radical cation by standard antioxidants in a linear fashion (Figure 6) shows the potential of the method for the determination of the
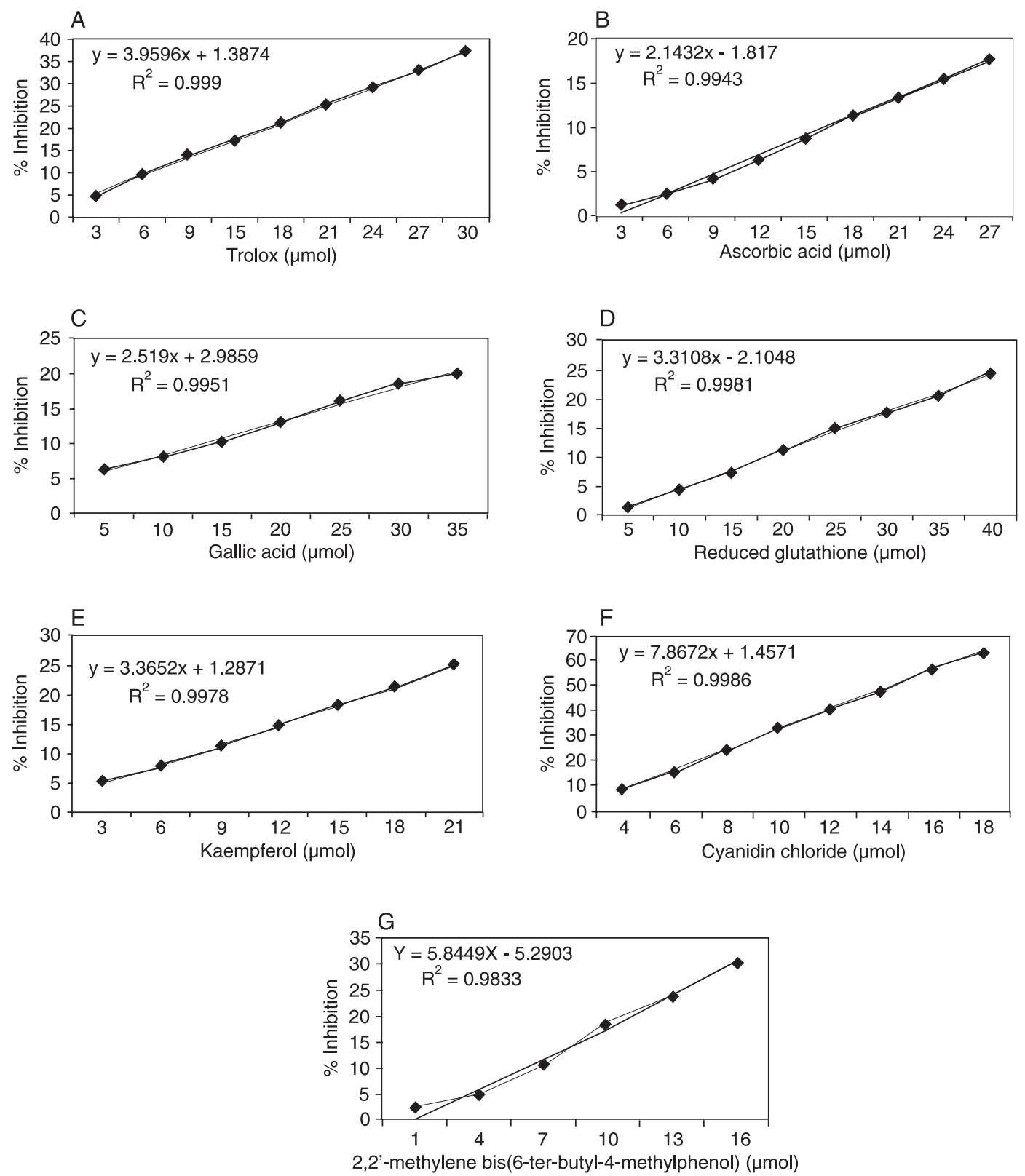

Figure 6. Effects of antioxidant concentration on the inhibition of trifluoperazine dihydrochloride. $A$, Trolox; $B$, ascorbic acid; $C$, gallic acid; $D$, reduced glutathione; $E$, kaempferol; $F$, cyanidin chloride; $G$, 2,2'-methylene bis(6-ter-butyl-4-methylphenol). 
antioxidant activity of different biological systems.

The reaction of standard antioxidants such as trolox, ascorbic acid, gallic acid, etc., with TFPH has been found to be rapid and reproducible, with the end point reading becoming stable practically within one min, showing the completion of the reaction. Figure 7 shows the kinetics of the reaction between specific antioxidants and the TFPH radical cation at $502 \mathrm{~nm}$. The results show that the reaction with the TFPH radical cation was completed within $1 \mathrm{~min}$.

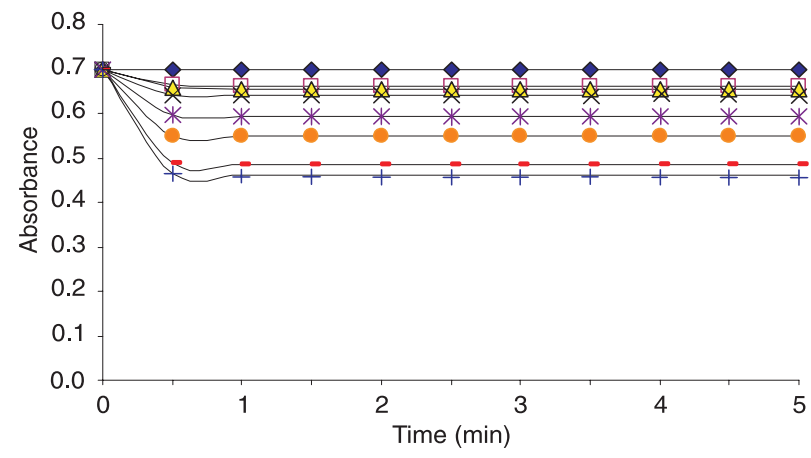

$\checkmark$ Unscavenged TFPH radical cation $\square$ Trolox (3 mM)

$\triangle$ Ascorbic acid (12 mM) $\quad \times$ Gallic acid $(10 \mathrm{mM})$

$*$ Reduced glutathione $(25 \mathrm{mM}) \quad-$ - Kaempferol $(18 \mathrm{mM})$

+ Cyanidin chloride $(10 \mathrm{mM})$

- 2,2'-methylene bis

(6-ter-butyl-4-methylphenol)

Figure 7. Effect of time on the scavenging of trifluoperazine dihydrochloride (TFPH) radical cation with the addition of standard antioxidants.

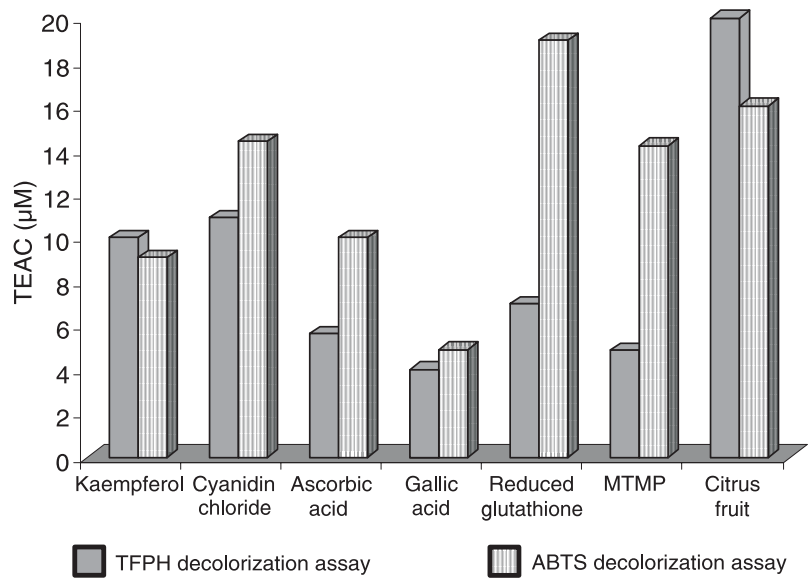

Figure 8. Comparison of antioxidant activity as trolox equivalent antioxidant capacity (TEAC; $\mu \mathrm{M})$ of standard antioxidants (12 $\mu \mathrm{M}$ kaempferol; $6 \mu \mathrm{M}$ cyanidin chloride; $8 \mu \mathrm{M}$ ascorbic acid; 30 $\mu \mathrm{M}$ gallic acid; $15 \mu \mathrm{M}$ reduced glutathione; $5 \mu \mathrm{M}$ 2,2'-methylene bis(6-ter-butyl-4-methyl phenol (MTMP); $10 \mu \mathrm{L}$ citrus fruit) determined by trifluoperazine dihydrochloride (TFPH) and 2,2' azinobis-[3-ethylbenzthiazoline-6-sulfonic acid] (ABTS) decolorization assays.
Antioxidants exist as different molecular species depending upon the nature and the $\mathrm{pH}$ of the medium. Recently, anthocyanins have been reported to exist in four different molecular forms in aqueous medium where the relative proportion of these forms depends upon the $\mathrm{pH}$ of the medium (13). Since the extent of solubilization/stabilization of different antioxidant forms varies with $\mathrm{pH}$ (14) and different human body fluids have different $\mathrm{pHs}$, each exogenous antioxidant has a unique net antioxidant capacity in vivo which may be quite different from that which is measured even with the most sophisticated decolorization assay in vitro. Thus, while assessing the antioxidant power of different antioxidants, along with other factors, the $\mathrm{pH}$ of their natural site of occurrence and at the place of their action in vivo should be taken into account. Generally the TEAC values reported in the literature are given at physiological pH 7.4 (21-23) which in the case of phytochemicals represent the antioxidant potential of various deprotonated states at different molar ratios. Thus, the claim that almost equal or comparable results are obtained by employing a variety of assays at different pH's might be questioned. The present assay ensures the measurement of the "intrinsic antioxidant activity" of a range of antioxidants in the non-deprotonated state of a single species by allowing the radical cation and the antioxidants (in a single nondeprotonated state) to react at low $\mathrm{pH}$.

A comparison of the TEAC values of antioxidants obtained by the ABTS persulfate decolorization assay and the TFPH persulfate decolorization assay is shown in Figure 8. It is obvious from the figure that the values from the two assays are comparable for kaempferol, cyanidin chloride, gallic acid, and citrus fruits, and differ quite reasonably for the remaining antioxidants. The TFPH assay has proven to be highly reproducible. Replicate measures from both assays showed a within assay mean percent relative standard deviation below $0.5 \%$ for standard antioxidants used.

At the same time, the newly developed TFPH decolorization assay is equally applicable for the determination of both hydrophilic and lipophilic antioxidants. The approximately $50 \%$ higher TEAC value (ABTS decolorization assay) obtained for cyanidin chloride is in agreement with the claim that the radical scavenging capacity of anthocyanins increases with increasing $\mathrm{pH}$ (24).

In addition to determining the intrinsic antioxidant activity, the proposed assay is less expensive (costs about US\$4 per 100 assays) and requires only 20 min for preparation of radical cation solution in comparison with ABTS assay in which almost $12-16 \mathrm{~h}$ are required for preparation of a stable ABTS radical cation solution. 


\section{References}

1. Baynes JW. Role of oxidative stress in development of complications in diabetes. Diabetes 1991; 40: 405-412.

2. Halliwell B, Gutteridge JMC. Free radicals in biology and medicine. 2nd edn. Oxford: Oxford University Press; 1989.

3. Yagi K. Lipid peroxides and human diseases. Chem Phys Lipids 1987; 45: 337-351.

4. Yla-Herttuala S, Palinski W, Rosenfeld ME, Parthasarathy $S$, Carew TE, Butler S, et al. Evidence for the presence of oxidatively modified low density lipoprotein in atherosclerotic lesions of rabbit and man. J Clin Invest 1989; 84: 10861095.

5. Ames BN, Shigenaga MK. Oxidants are a major contributor to aging. Ann NY Acad Sci 1992; 663: 85-96.

6. Folin $\mathrm{O}$, Ciocalteu V. On tyrosine and tryptophane determinations in proteins. J Biol Chem 1927; 73: 627-650.

7. Singleton VL, Orthofer RM, Ramuela-Raventos RM. Analysis of total phenols and other oxidation substrates and antioxidants by means of Folin-Ciocalteu reagent. Methods Enzymol 1999; 299: 152-178.

8. Miller NJ, Rice-Evans C, Davies MJ, Gopinathan V, Milner A. A novel method for measuring antioxidant capacity and its application to monitoring the antioxidant status in premature neonates. Clin Sci 1993; 84: 407-412.

9. Re R, Pellegrini N, Proteggente A, Pannala A, Yang M, Rice-Evans C. Antioxidant activity applying an improved ABTS radical cation decolorization assay. Free Radic Biol Med 1999; 26: 1231-1237.

10. Benzie IF, Strain JJ. The ferric reducing ability of plasma (FRAP) as a measure of "antioxidant power": the FRAP assay. Anal Biochem 1996; 239: 70-76.

11. Pulido R, Bravo L, Saura-Calixto F. Antioxidant activity of dietary polyphenols as determined by a modified ferric reducing/antioxidant power assay. J Agric Food Chem 2000; 48: 3396-3402.

12. Fogliano V, Verde V, Randazzo G, Ritieni A. Method for measuring antioxidant activity and its application to monitoring the antioxidant capacity of wines. J Agric Food Chem 1999; 47: 1035-1040.

13. Borkowski T, Szymusiak H, Gliszczynska-Rwiglo A, Rietjens IM, Tyrakowska B. Radical scavenging capacity of wine anthocyanins is strongly pH-dependent. J Agric Food Chem 2005; 53: 5526-5534.

14. van Acker SA, de Groot MJ, van den Berg DJ, Tromp MN, Donne-Op den Kelder G, van der Vijgh WJF, et al. A quantum chemical explanation of the antioxidant activity of flavonoids. Chem Res Toxicol 1996; 9: 1305-1312.

15. Lemanska K, Szymusiak H, Tyrakowska B, Zielinski R, Soffers AE, Rietjens IM. The influence of $\mathrm{pH}$ on antioxidant properties and the mechanism of antioxidant action of hydroxyflavones. Free Radic Biol Med 2001; 31: 869-881.

16. Cotzias GC, Borg DC, Hughes ER, Bertinchamps A, Papavasiliou PS. Phenothiazines: curative or causative in regard to parkinsonism? Rev Can Biol 1961; 20: 289-294.

17. Cotzias GC, Borg DC. Association for research in nervous and mental disease. Proceedings. Baltimore: Williams and Wilkens; 1962.

18. Michaelis L, Schubert MP, Granick S. Semiquinone radicals of the thiazines. J Am Chem Soc 1940; 62: 204-211.

19. Nakajima H. Studies on amine oxidase: effects of chlorpromazine and its analogues. J Biochem 1959; 46: 1675.

20. Borg DC, Cotzias GC. Interaction of trace metals with phenothiazine drug derivatives. I. Structure-reactivity correlations. Proc Natl Acad Sci USA 1962; 48: 617-623.

21. Rice-Evans CA, Miller NJ, Paganga G. Structure-antioxidant activity relationships of flavonoids and phenolic acids. Free Radic Biol Med 1996; 20: 933-956.

22. Rice-Evans CA, Miller NJ, Paganga G. Antioxidant properties of phenolic compounds. Trends Plant Sci 1997; 2: 152158.

23. Rice-Evans CA, Miller NJ, Bolwell PG, Bramley PM, Pridham JB. The relative antioxidant activities of plant-derived polyphenolic flavonoids. Free Radic Res 1995; 22: 375-383.

24. Ozgen M, Reese RN, Tulio AZ Jr, Scheerens JC, Miller AR. Modified 2,2-azino-bis-3-ethylbenzothiazoline-6-sulfonic acid (ABTS) method to measure antioxidant capacity of selected small fruits and comparison to ferric reducing antioxidant power (FRAP) and 2,2'-diphenyl-1-picrylhydrazyl (DPPH) methods. J Agric Food Chem 2006; 54: 1151-1157. 